LA-UR-01-5243

Approved for public release; distribution is unlimited.

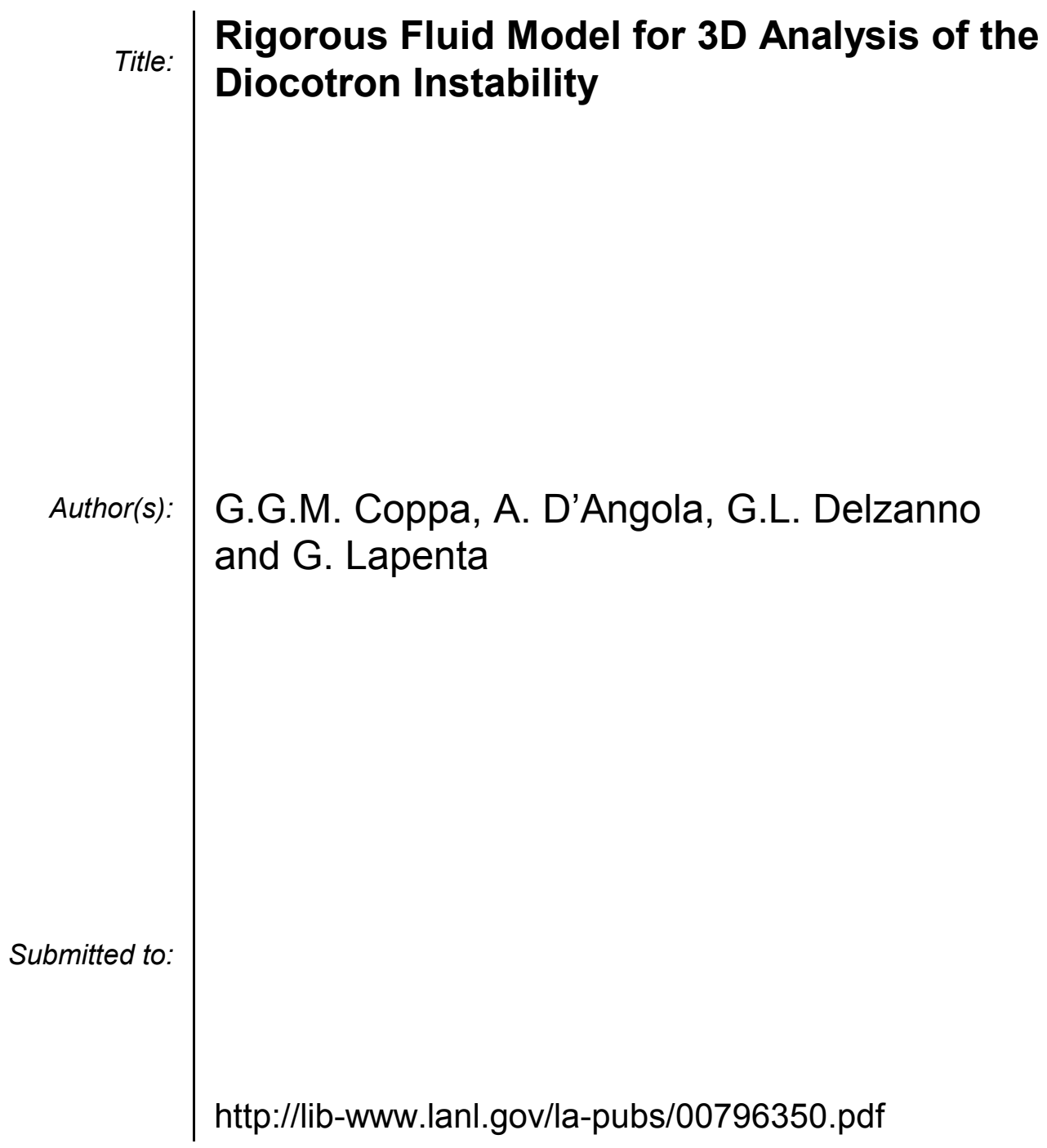

Los Alamos National Laboratory, an affirmative action/equal opportunity employer, is operated by the University of California for the U.S. Department of Energy under contract W-7405-ENG-36. By acceptance of this article, the publisher recognizes that the U.S. Government retains a nonexclusive, royaltyfree license to publish or reproduce the published form of this contribution, or to allow others to do so, for U.S. Government purposes. Los Alamos National Laboratory requests that the publisher identify this article as work performed under the auspices of the U.S. Department of Energy. Los Alamos National Laboratory strongly supports academic freedom and a researcher's right to publish; as an institution, however, the Laboratory does not endorse the viewpoint of a publication or guarantee its technical correctness. 


\title{
Rigorous Fluid Model for 3D Analysis of the Diocotron Instability
}

\author{
G.G.M. Coppa*, A. D’Angola*, G.L. Delzanno* and G. Lapenta ${ }^{\dagger}$ \\ ${ }^{*}$ Istituto Nazionale di Fisica per la Materia (INFM) and Dipartimento di Energetica, Politecnico di \\ Torino, Corso Duca degli Abruzzi 24, 10129 Torino, Italy \\ ${ }^{\dagger}$ Istituto Nazionale di Fisica per la Materia (INFM) and Theoretical Division, Los Alamos \\ National Laboratory, Los Alamos, NM 87545, USA
}

\begin{abstract}
A refinement to the theory published by Finn et al. is presented here. Compression effects are taken into account by a rigorous definition of the plasma length and by modifying the expression of the velocity field. The perturbation of the plasma length is calculated exactly by a suitable Green function. Growth rates and real frequencies of the unstable $m_{\theta}=1$ mode are compared with the experimental values, showing a good agreement when compression effects are strong (i.e., for short traps).
\end{abstract}

\section{INTRODUCTION}

The $m_{\theta}=1$ diocotron instability is one of the open problems in non-neutral plasma theory. According to the classical theory, the $m_{\theta}=1$ diocotron spectrum does not present discrete unstable modes (regardless of the equilibrium density profile) [1], while the continuum spectrum can only produce an algebraic growth proportional to $t^{1 / 2}$ [2]. On the contrary, experiments show that the linear growth of the mode is exponential $[3,4]$.

Finn et al. [5] provided a justification of the $m_{\theta}=1$ diocotron instability in terms of compression of the plasma column due to the confining potential along the axial direction of the trap. However, the theory by Finn et al. neglects the finite electron temperature and the effects of the perturbation of the plasma length in the axial direction are treated in a simplified manner. These two issues are treated rigorously in a recent work by the Authors [6].

In particular, the new model differs from the one in Ref. [5] for three main aspects:

1. a rigorous definition of the length of the plasma column is used;

2. an effective electrostatic potential is introduced, in order to calculate the $\mathbf{E} \times \mathbf{B}$ drift on a string of variable axial density (finite temperature effects are included);

3. the perturbation of the plasma length, induced by density variations, is calculated in terms of a suitable Green function.

In the present work the theory presented in Ref. [6] is reviewed and comparisons with experimental data provided by Kabantsev and Driscoll [4] are presented. 


\section{THE PHYSICAL MODEL}

A Penning trap confining an electron plasma is considered in the following. The central electrode, which extends between $z=-L_{c} / 2$ and $z=L_{c} / 2$, is grounded, while the end electrodes (each having length $L_{s}$ ) are at a negative potential, $-V$. The radius of the trap is indicated by $R_{\mathrm{W}}$ and the gap between the central and the end electrodes is $L_{g}$.

The main difference of the present model with respect to the classical one is regarding electrons as string of variable length and, consequently, of variable density (while in the classical approach they are considered as strings of uniform density). Since the plasma length changes radially (due to the confining potential), the columns are compressed or rarefied as they move radially. This phenomenon is known as compression effect.

The model can be deduced starting from the following two hypotheses:

1. Due to the presence of an axial magnetic field, in the characteristic time scale of the diocotron instability the Maxwell-Boltzmann distribution is reached only along the $z$ direction [7] and the particle density is

$$
n(r, \theta, z, t)=n_{c}(r, \theta, t) \exp \left[\frac{\phi(r, \theta, z, t)-\phi_{c}(r, \theta, t)}{\Theta}\right]
$$

where $\Theta=k T / e$ is the electron temperature expressed in Volts, while $n_{c}(r, \theta, t)$ and $\phi_{c}(r, \theta, t)$ are respectively the electron density and the electrostatic potential in the central region of the trap, i.e., for $|z|<L_{c} / 2$.

2. Electrons move in the transverse plane due to the $\mathbf{E} \times \mathbf{B}$ drift velocity:

$$
\mathbf{v}_{\perp}(r, \theta, z, t)=\frac{1}{B_{0}} \mathbf{E}_{\perp}(r, \theta, z, t) \times \widehat{\mathbf{e}}_{z}
$$

\section{The continuity equation}

Instead of $n_{c}(r, \theta, t)$, experiments yield the line integrated density, $\sigma(r, \theta, t)$, given by

$$
\sigma(r, \theta, t)=\int_{-\infty}^{+\infty} n(r, \theta, z, t) d z=n_{c}(r, \theta, t) \mathcal{L}(r, \theta, t),
$$

where the effective length of the plasma column, $\mathcal{L}(r, \theta, t)$, has been defined as

$$
\mathcal{L}(r, \theta, t)=\int_{-\infty}^{+\infty} \exp \left[\frac{\phi(r, \theta, z, t)-\phi_{c}(r, \theta, t)}{\Theta}\right] d z .
$$

Averaging the continuity equation for $n(r, \theta, z, t)$ over $z$, one obtains a new continuity equation for the line integrated density

$$
\frac{\partial \sigma}{\partial t}+\nabla_{\perp} \cdot\left(\mathbf{V}_{\perp} \sigma\right)=0
$$

with the velocity given by a suitable $z$-averaged $\mathbf{E} \times \mathbf{B}$ drift. 


\section{The $z$-averaged $\mathbf{E} \times \mathbf{B}$ drift}

The present theory differs from the one by Finn et al. [5] in the evaluation of $\mathbf{V}_{\perp}$. In fact, the velocity is here calculated in terms of the $\mathbf{E} \times \mathbf{B}$ drift estimated on a whole string (a similar assumption has been employed by Fine and Driscoll [8]) and it is given by

$$
\mathbf{V}_{\perp}=\frac{1}{B_{0}} \frac{\int n \mathbf{E}_{\perp} d z}{\int n d z} \times \widehat{\mathbf{e}}_{z}=\frac{1}{B_{0}} \widehat{\mathbf{e}}_{z} \times \frac{\int \exp \left(\frac{\phi}{\Theta}\right) \nabla_{\perp} \phi d z}{\int \exp \left(\frac{\phi}{\Theta}\right) d z} .
$$

Equation (6) can be rewritten as

$$
\mathbf{V}_{\perp}=\frac{1}{B_{0}} \widehat{\mathbf{e}}_{z} \times \nabla_{\perp} \phi_{\mathrm{eff}},
$$

where the effective potential, $\phi_{\mathrm{eff}}(r, \theta, t)$, is defined as

$$
\phi_{\mathrm{eff}}=\phi_{c}+\Theta \log \frac{\mathcal{L}}{\mathcal{L}_{r=0}} .
$$

\section{The model}

The model is completed by the Poisson equation for $\phi$. The set of equations can be written in a more compact way by defining the quantity $\Psi(r, \theta, z, t)=\phi(r, \theta, z, t)-$ $\phi_{c}(r, \theta, t)$, which represents the correction to the potential in a trap of finite length, with respect to the one in a trap of infinite length. Finally, the following system of equations is to be solved:

$$
\left\{\begin{array}{l}
\frac{\partial \sigma}{\partial t}=-\mathbf{V}_{\perp} \cdot \nabla_{\perp} \sigma \\
\mathbf{V}_{\perp}=\frac{1}{B_{0}} \widehat{\mathbf{e}}_{z} \times \nabla_{\perp} \phi_{\text {eff }} \\
\mathcal{L}=\int \exp \left(\frac{\Psi}{\Theta}\right) d z \\
\nabla^{2} \Psi=\frac{e}{\varepsilon_{0}} \frac{\sigma}{\mathcal{L}}\left[\exp \left(\frac{\Psi}{\Theta}\right)-1\right] \\
\nabla_{\perp}^{2} \phi_{c}=\frac{e}{\varepsilon_{0}} \frac{\sigma}{\mathcal{L}} \\
\phi_{\text {eff }}=\phi_{c}+\Theta \log \frac{\mathcal{L}}{\mathcal{L}_{r=0}}
\end{array} .\right.
$$


TABLE 1. Confining potential and geometrical parameters of the experiments performed by Kabantsev and Driscoll [4].

\begin{tabular}{rrrrr}
\hline & $V[\mathbf{V}]$ & $L_{c}[\mathbf{c m}]$ & $L_{g}[\mathbf{c m}]$ & $L_{s}[\mathbf{c m}]$ \\
\hline $\mathbf{A}$ & 36 & 34.6 & 0.175 & 6.8 \\
$\mathbf{B}$ & 80 & 20.7 & 0.175 & 13.8 \\
$\mathbf{C}$ & 100 & 7 & 0.175 & 6.8 \\
\hline
\end{tabular}

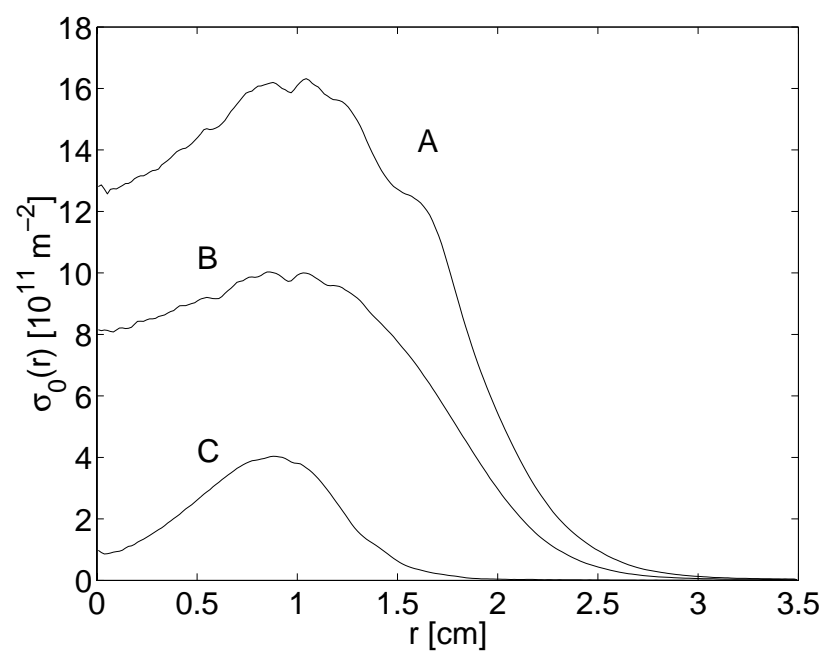

FIGURE 1. Experimental line integrated density from experiments by Kabantsev and Driscoll [4].

A modified Rayleigh criterion for linear stability and the conservation of canonical angular momentum and of the energy of the system can be deduced from system (9) [6]. The criterion proves that when $\sigma_{0}(r)$ is a monotonic function, the system is linearly stable.

\section{COMPARISON WITH EXPERIMENTAL RESULTS}

Growth rates calculated with the present theory have been compared with those resulting from recent experiments performed by Kabantsev and Driscoll [4]. For each experiment the electron temperature is approximately $1 \mathrm{~V}$ and the axial magnetic field is $B_{0}=0.4 \mathrm{~T}$. Three different cases (labelled $A-B-C$ ) have been considered. Table 1 summarizes the confining voltage and the geometrical parameters of the trap for these cases.

For each case, the initial $z$-integrated density, $\sigma_{0}(r)$, is reported in Fig. 1. From such measured density distributions, the corresponding profiles for $n_{c}(r)$ and $\mathcal{L}(r)$ have been calculated, by solving Eqs. (9) iteratively for the initial equilibrium. The results of the calculations are presented in Figs. 2 and 3. Growth rates of the instability, $\gamma$, and real frequencies, $f$, for the $m_{\theta}=1$ mode have been evaluated by linearizing system (9). Table 2 summarizes the results. 


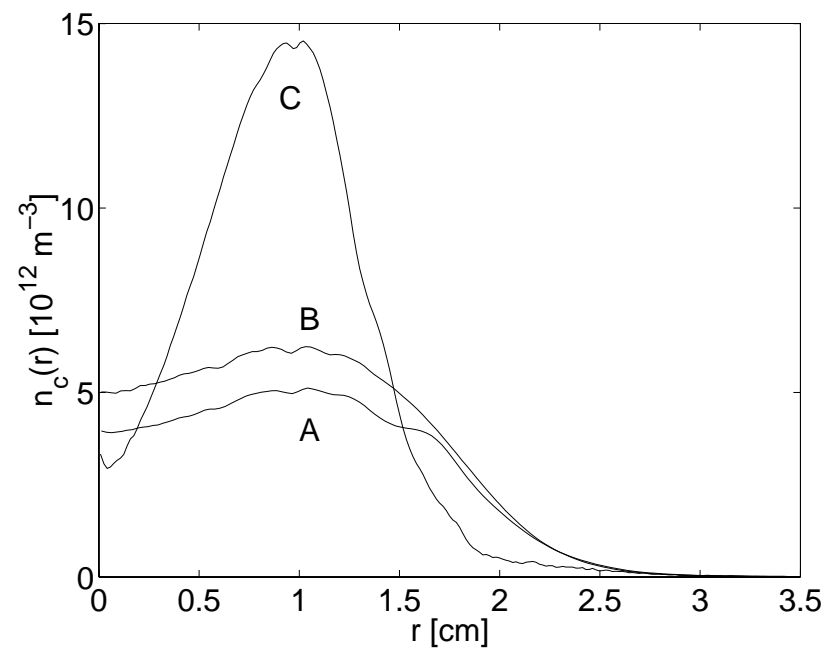

FIGURE 2. Equilibrium density profile calculated from experimental $\sigma_{0}$.

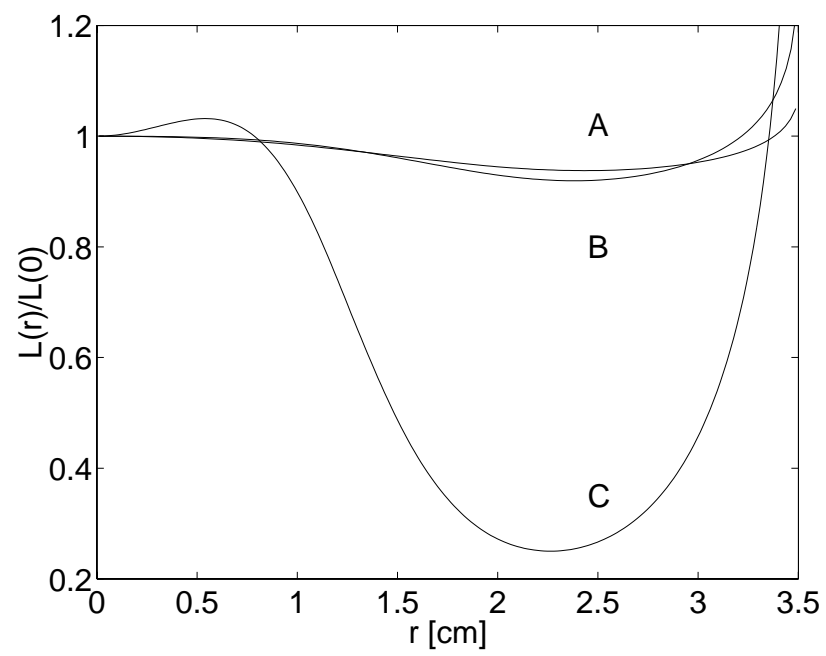

FIGURE 3. Equilibrium length profile calculated from experimental $\sigma_{0}$.

A few considerations are in order. It can be noticed that in a short trap (case $C$ ) compression effects are very strong and the equilibrium length profile varies considerably with $r$. On the contrary, for longer traps (cases $A$ and $B$ ) the equilibrium length profile has a small variation with respect to its value for $r=0$ (10\% inside the plasma radius). When compression effects are small (i.e., for long traps), the growth rates calculated with the present theory are not comparable with the corresponding experimental values. Viceversa, when compression effects are strong (i.e., for short traps) the agreement between theory and experiment is remarkable. When compression effects are small, the disagreement between theory and experiment is probably to be explained in terms of kinetic effects, as pointed out in Refs. [9] and [10]. 
TABLE 2. Real frequency and growth rate (in $\mathrm{kHz}$ ) for the unstable $m_{\theta}=1$ mode

\begin{tabular}{ccccccc}
\hline & \multicolumn{3}{c}{ experiment } & \multicolumn{3}{c}{ theory } \\
& $f$ & $\gamma$ & $\gamma / f$ & $f$ & $\gamma$ & $\gamma / f$ \\
\hline A & 17.9 & 6.0 & 0.34 & 17.0 & 0.99 & 0.058 \\
\hline B & 23.2 & 5.6 & 0.24 & 21.0 & 0.93 & 0.044 \\
\hline C & 42.7 & 10.6 & 0.25 & 36.1 & 8.5 & 0.24 \\
\hline
\end{tabular}

\section{ACKNOWLEDGMENTS}

The authors gratefully thank Andrey Kabantsev and Fred Driscoll for providing experimental data used in the present work. The authors are also grateful to John Finn and Diego del Castillo-Negrete for stimulating discussions about the linear theory of the $m_{\theta}=1$ mode and to Thomas O'Neil, Daniel Dubin, David Schecter and Terry Hilsabeck for their useful suggestions.

Work performed with a partial support by the Italian National Group for Mathematical Physics (GNFM-INDAM, research project: "Kinetic Theory and Fluid Models for NonNeutral Plasmas") and by the USA Department of Energy under contract W-7405-ENG36.

\section{REFERENCES}

1. Davidson, R. C., An Introduction to the Physics of Nonneutral Plasmas, Addison-Wesley, Redwood City, 1990.

2. Smith, R. A., and Rosenbluth, M. N., Phys. Rev. Lett. 64, 649-652 (1990).

3. Driscoll, C. F., and Fine, K. S., Phys. Fluids B 2, 1359-1366 (1990).

4. Kabantsev, A. A., and Driscoll, C. F., "End Shape Effects on the $m_{\theta}=1$ Diocotron Instability in Hollow Electron Columns" in 1999 Workshop on Nonneutral Plasmas, edited by J. J. Bollinger, R. L. Spencer, R. C. Davidson, AIP Conference Proceedings 498, New York, 1999, pp. 208-213.

5. Finn, J. M., del-Castillo-Negrete, D., and Barnes, D. C., Phys. Plasmas 6, 3744-3758 (1999); Finn, J. M., del-Castillo-Negrete, D., and Barnes, D. C., Phys. Rev. Lett. 84, 2401-2404 (2000).

6. Coppa, G. G. M., D’Angola, A., Delzanno, G. L., and Lapenta, G., Phys. Plasmas 8, 1133-1141 (2001).

7. Dubin, D. H. E., and O'Neil, T. M., Phys. Plasmas 5, 1305-1314 (1998).

8. Fine, K. S., and Driscoll, C. F., Phys. Plasmas 5, 601-607 (1998).

9. Hilsabeck, T. J., and O'Neil, T. M., Phys. Plasmas 8, 407-422 (2001).

10. Coppa, G. G. M., and Ricci, P., "Non-Collisional Kinetic Model for Non-Neutral Plasmas in a Penning Trap: General Properties and Stationary Solutions", these proceedings. 